\title{
Level-1 Data Driver Card of the ATLAS New Small Wheel Upgrade
}

\author{
Panagiotis Gkountoumis \\ National Technical University of Athens
}

On behalf of the ATLAS Muon Collaboration

MOCAST, 14-15 MAY 2015, Athens

$15 / 05 / 2015$

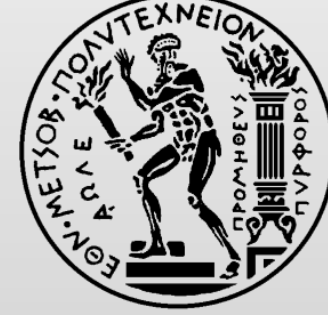




\section{Overview}

- CERN \& ATLAS experiment

- NSW electronics

- L1DDC

- General description

- Connectivity

- Rates

- Components

- Placement

- L1DDC prototype 1 \& 2

- Summary 


\section{CERN \& ATLAS experiment}

- CERN - largest particle physics laboratory (Franco-Swiss borders near Geneva)

- Large Hardon Collider - largest and most powerful particle accelerator

- $27 \mathrm{Km}$ ring, $100 \mathrm{~m}$ under surface

- 4 experiments (ALICE, ATLAS, CMS, LHCB)

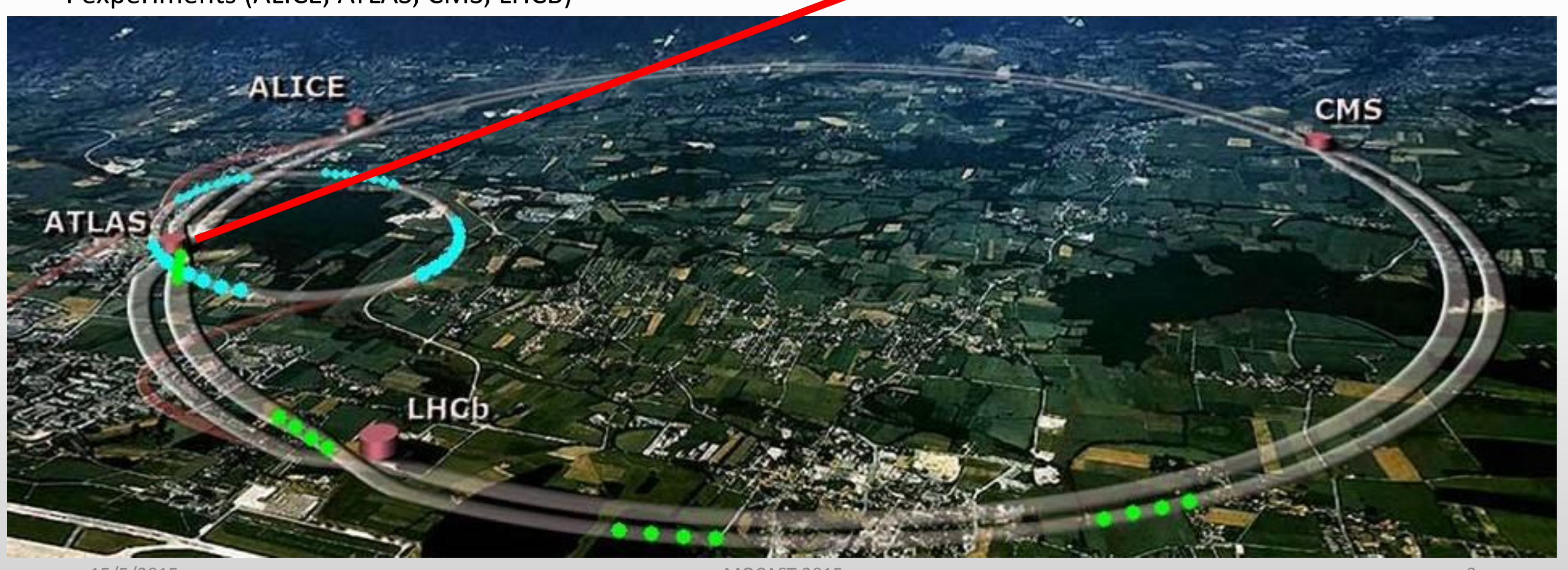




\section{The ATLAS experiment}

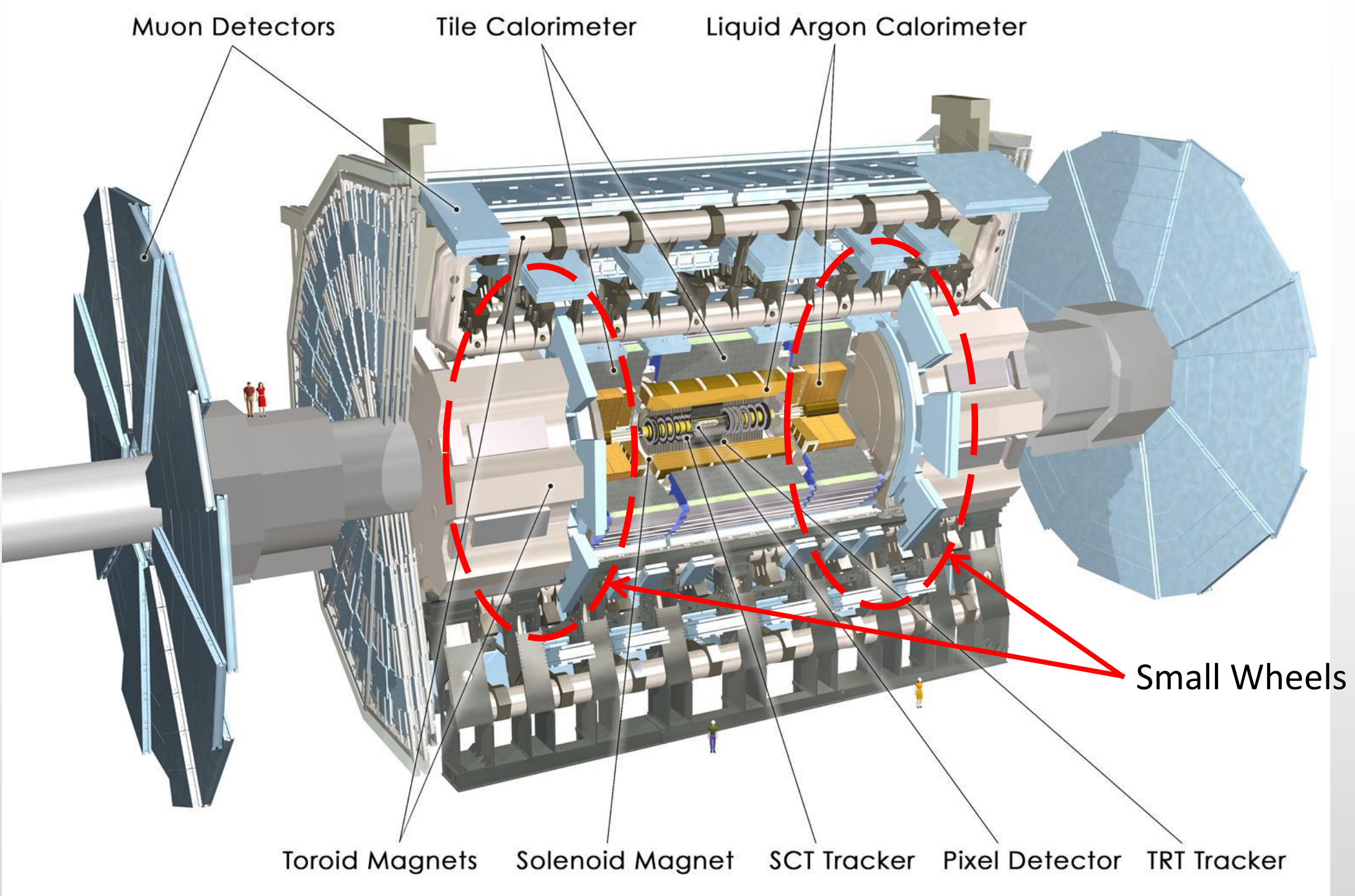

- ATLAS - General purpose detector

- Small Wheels are located between end-cap calorimeter and end-cap toroid

- Consist of CSC, TGC, MDT detectors

- $10 \mathrm{~m}$ in diameter

- High background rate - $L=2$ $5 \times 10^{34} \mathrm{~cm}^{-2} \mathrm{~s}^{-1}$ during LHC Run-3 and $\mathrm{HL}-\mathrm{LHC}$

- Particle rate will be increased

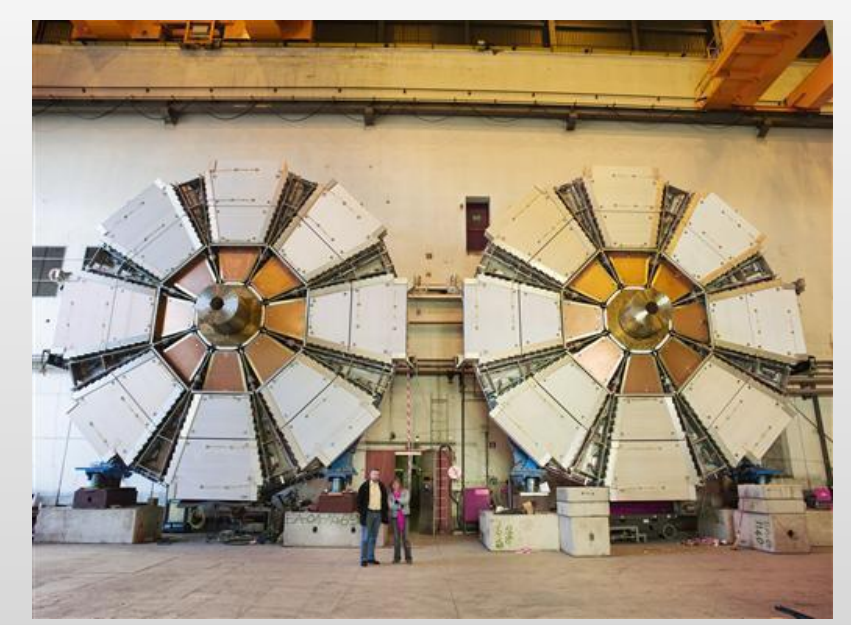




\section{New Small Wheel}

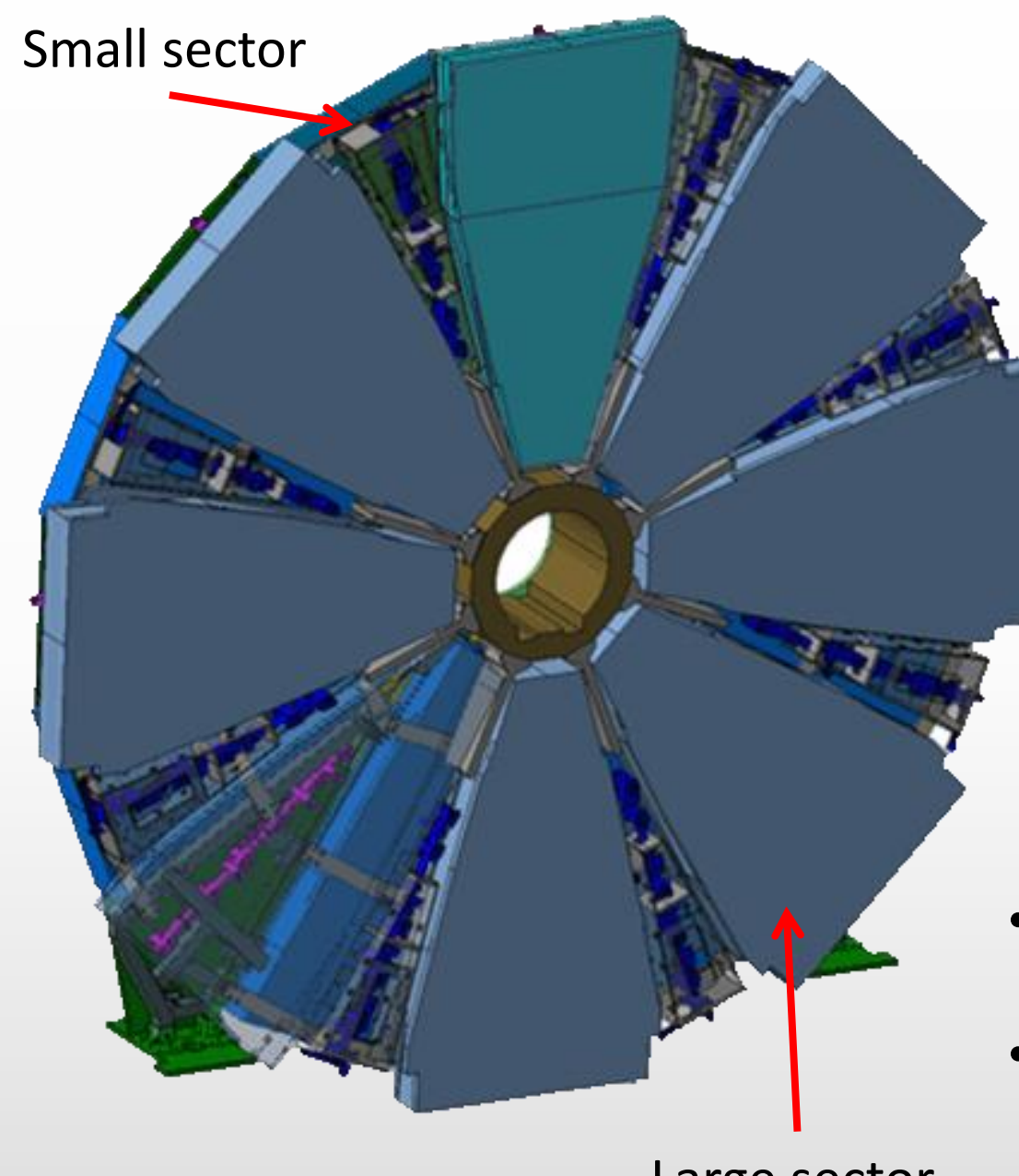

- NSW is a set of precision tracking and trigger detectors able to work at high rates

- 2 technologies sTGCs and micromegas detectors will provide tracking and trigger data

Large sector

- 8 small + 8 large sectors per wheel

- 8 sTGCs +8 micromegas planes per sector

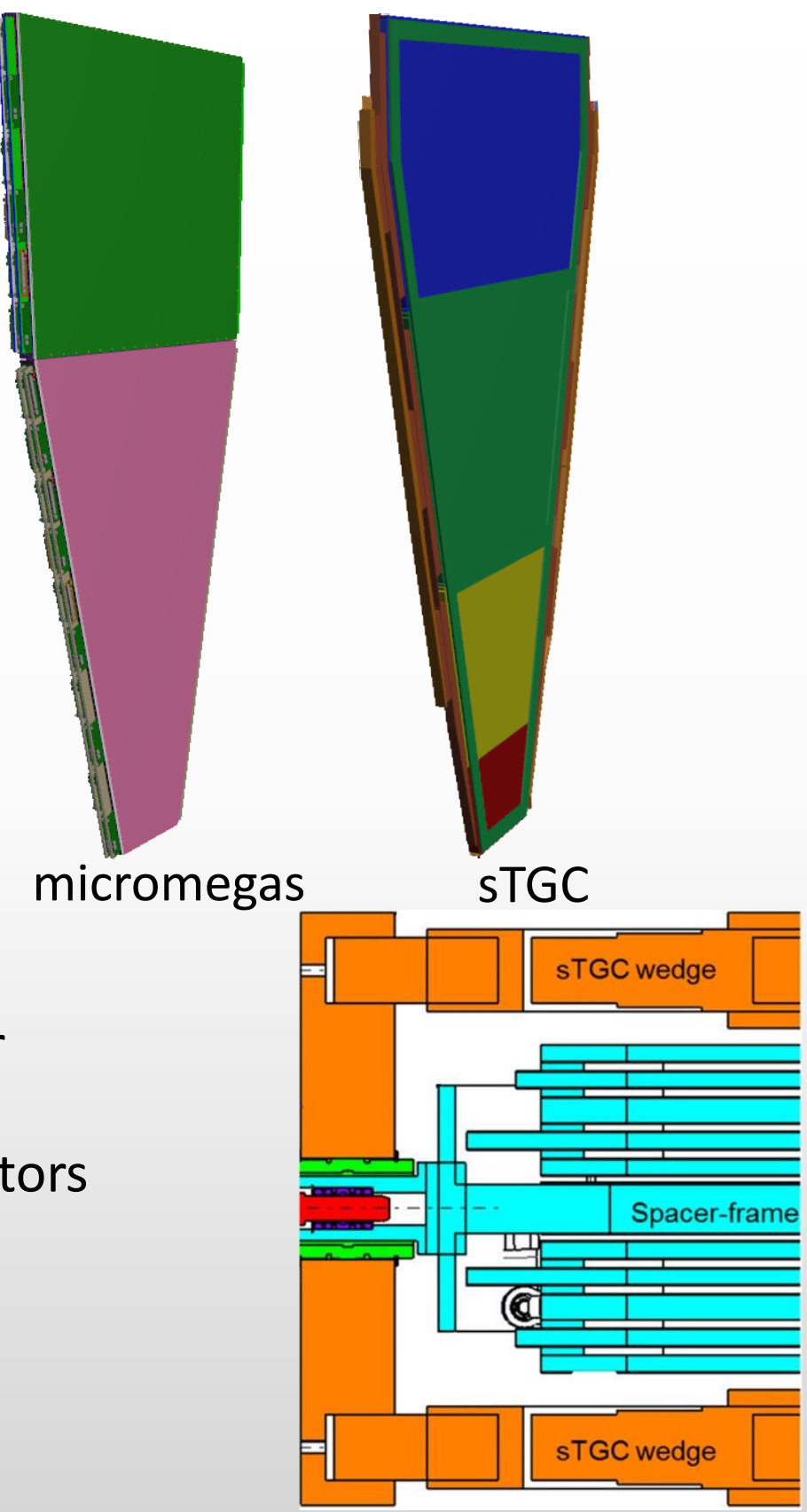




\section{NSW electronics trigger and dataflow}

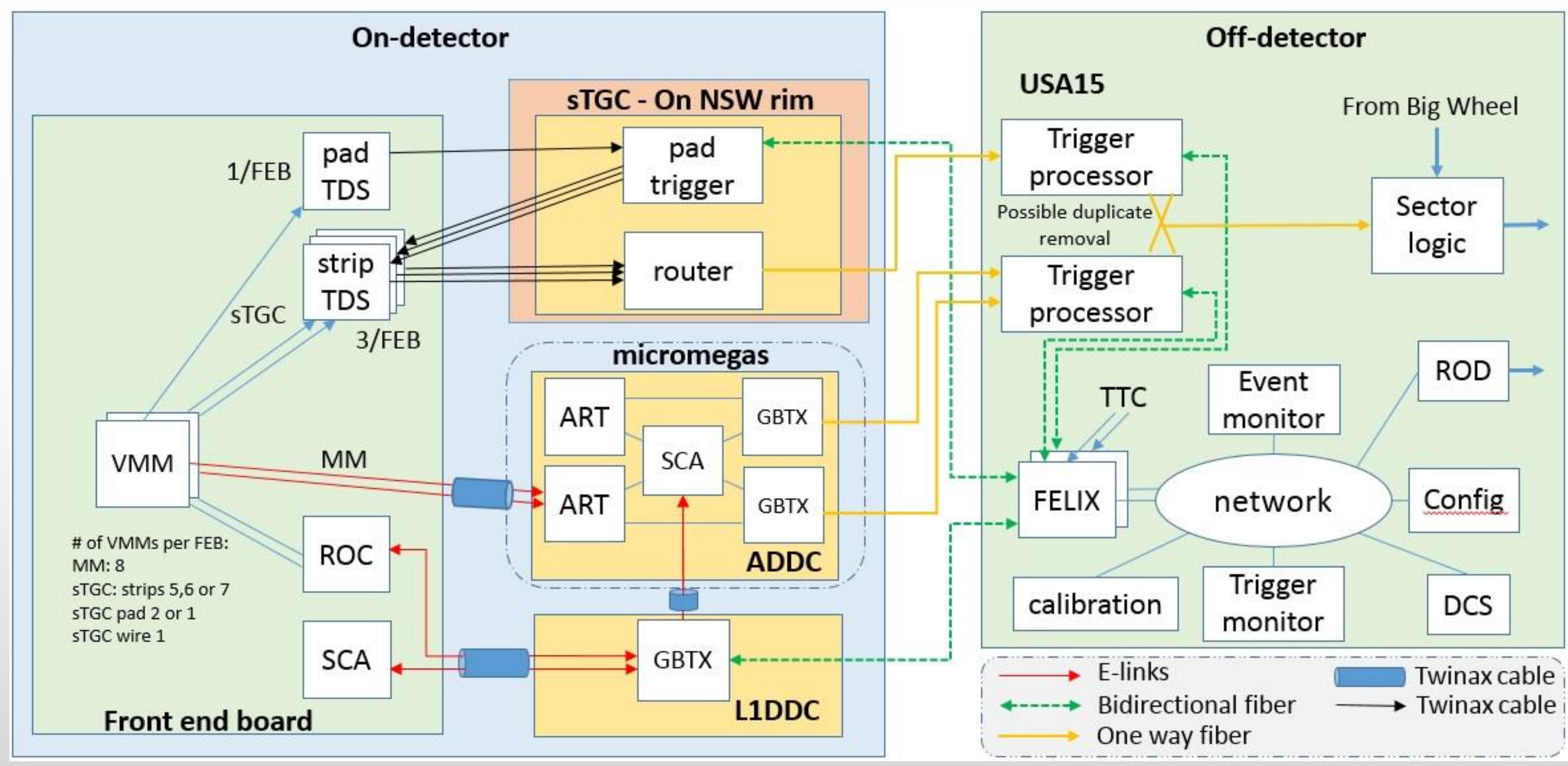




\section{L1DDC connectivity}

- 3 distinct paths to the front ends through one bidirectional link

\section{- DAQ data}

- Level-1 data (Time, charge and strip address)

- Configuration data

\section{- ASICS}

- GBTX

- GigaBit TransImpendence Amplifier (GBTIA) - Photo Diode

- GigaBit Laser Diode (GBLD)) - Laser

- Line rate $4.8 \mathrm{Gbps}$

- 1 frame of 120bits @ 40MHz (25ns)

- Header 4 bits

- Slow control 4 bits

- Data 80 bits

- FEC 32 bits (2 interleaved Reed-Solomon encoding)

- Error correction and error detection

- Up to 16 consecutive corrupted bits can be corrected

- User bandwidth 3.36Gbps

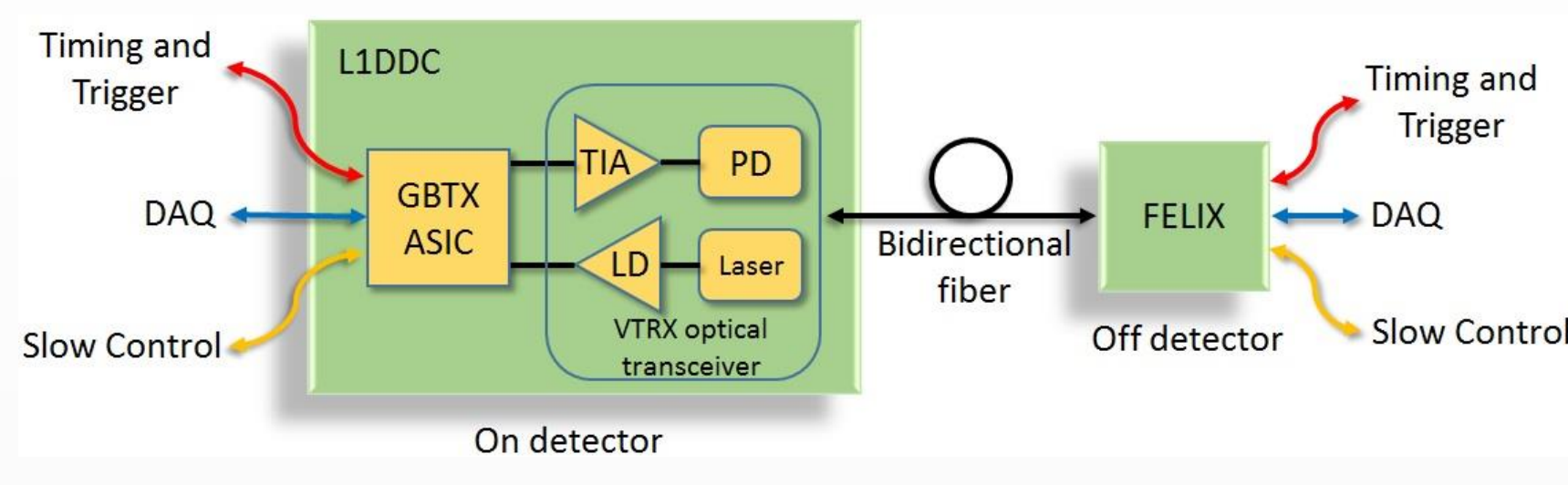




\section{GBTX ASIC Connectivity}

- 1 e-link has 3 differential pairs: Clock, Data out, Data in

- Up to 40 front-ends can be connected to 1 GBTx combined in 5 banks

- Each bank can support (programmable rate)

- 8 front ends @ 80Mbps

- 4 front ends @ 160Mbps

- 2 front ends @ 320Mbps

- Each bank can be configured at different rate

- 1 LIDDC is connected to 8 micromegas FEs

- 1 cable for FE to L1DDC connection

- 1 e-link for the ROC

- 1 e-link for the SCA

- 1 LIDDC is connected to 3 sTGC FEs

- 1 cable for FE to L1DDC connection

- 3 e-links for the ROC

- 1 e-link for the SCA

- Slow Control channel @ 80Mbps dedicated to

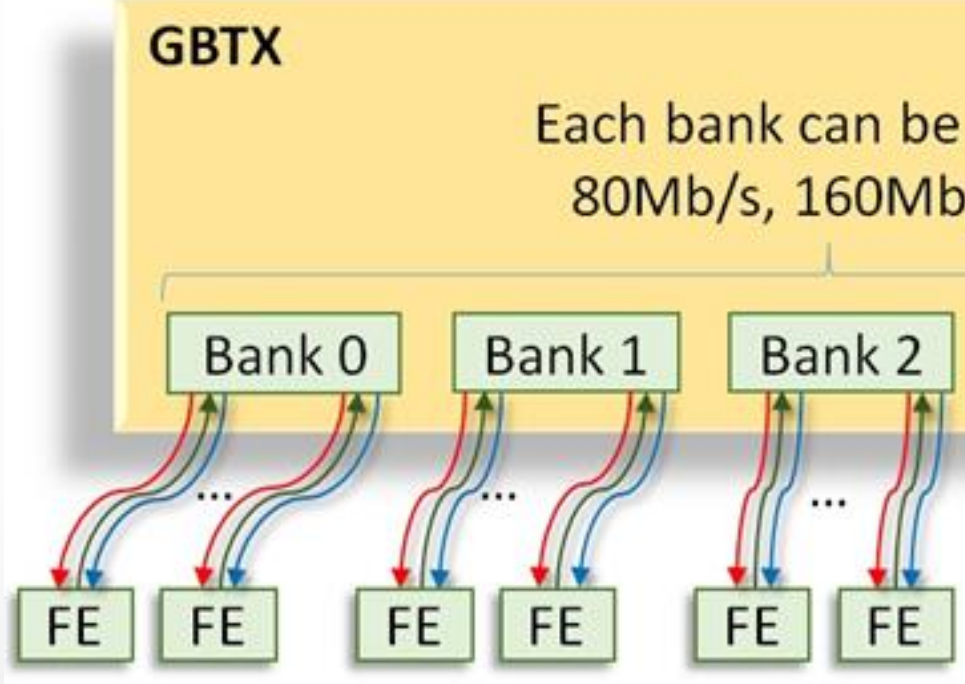

E-link (3 Differential pairs)

Clock $(\mathrm{Clk}+, \mathrm{Clk}-)$

Data out (Dout+, Dout-)

$\rightarrow$ Data in (Din+, Din-)
Slow Control at $80 \mathrm{Mb} / \mathrm{s}$ ADDC (1 e-link)

- E-links use Scalable Low-Voltage Signaling (SLVS), $\mathrm{Vcm}=0.2 \mathrm{~V}$, swing $200 \mathrm{mV}$

Banks

8FEs/Bank@80Mbps

4 FEs/Bank@160Mbps

2 FEs/Bank@320Mbps 


\section{L1DDC connectivity with the MMFE8 and ADDC boards}

Limits [Mbps]

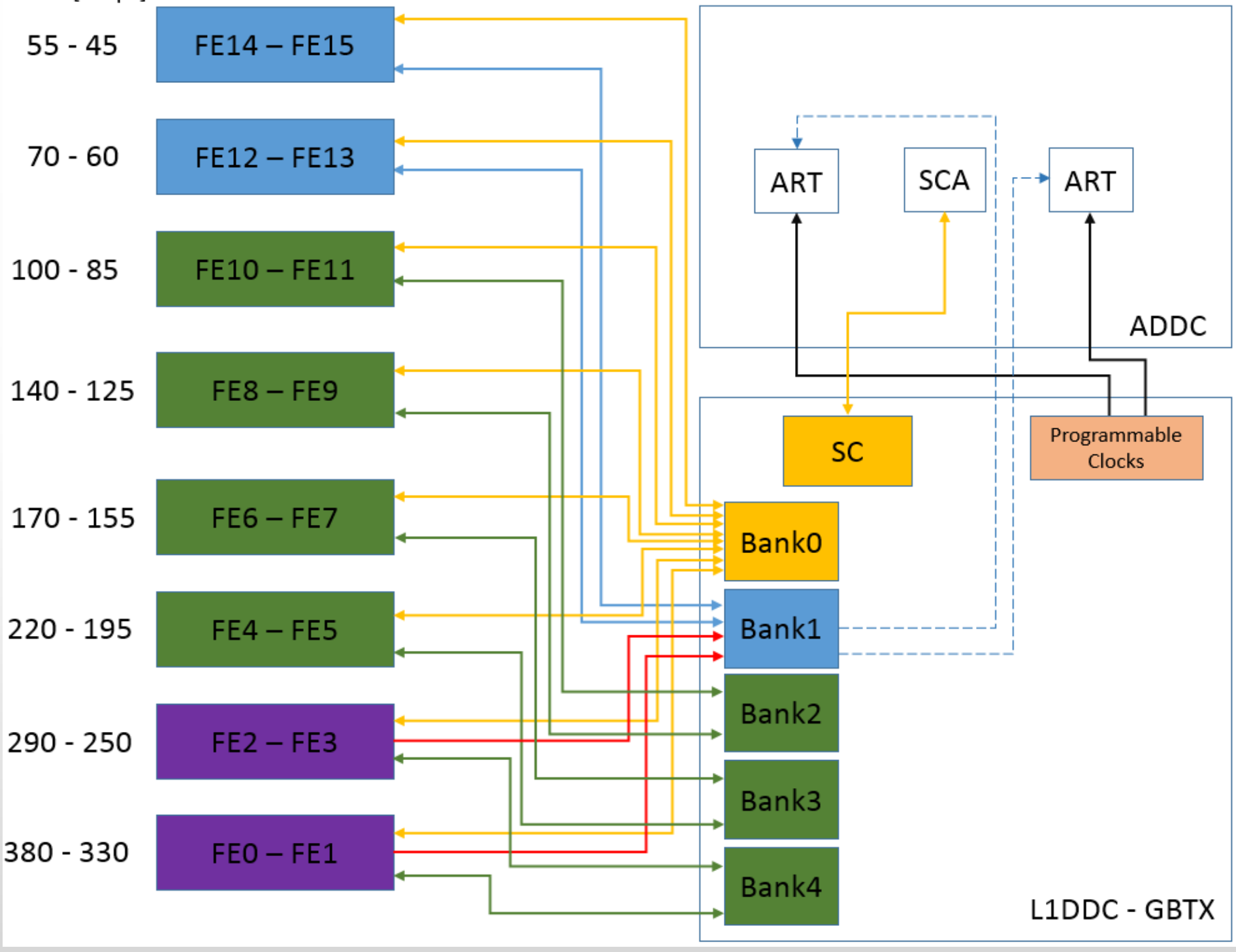

- SCA ASIC will be added to the ADDC board

- Slow Control channel of the GBTX will be used for the configuration of the SCA.

- 2 extra differential pairs (clocks) will be send from the L1DDC to the ADDC

- Either from the 8 programmable clocks of GBTX.

- $\quad$ Either form the two spares of the bank1.

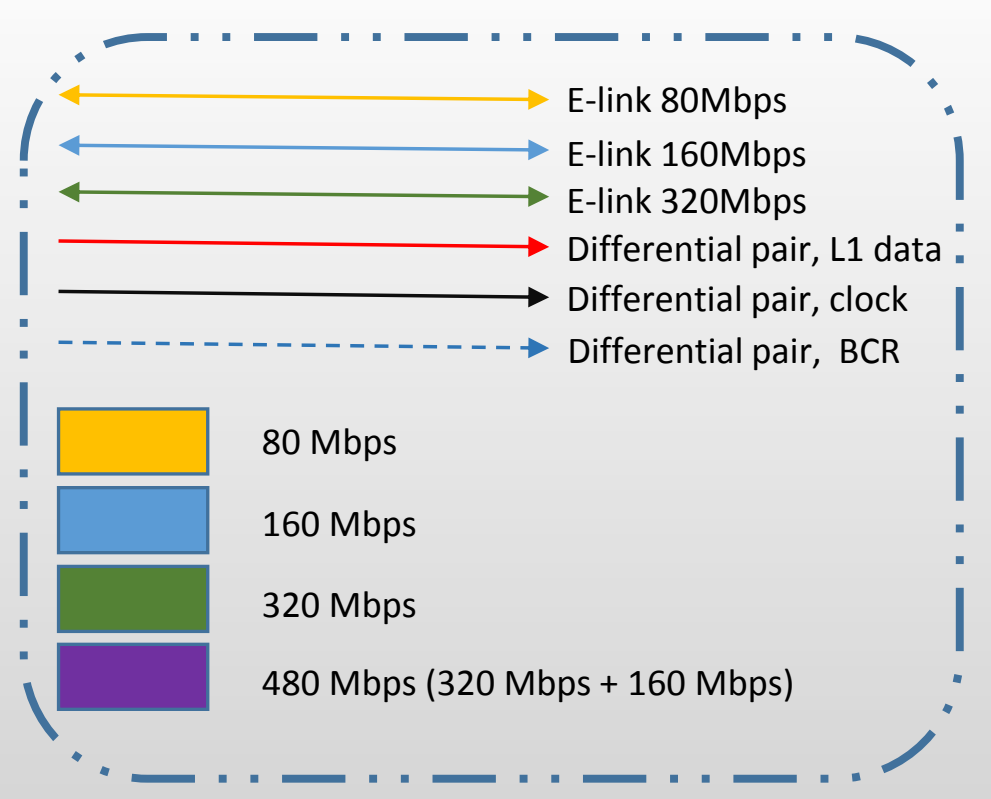




\section{L1DDC}

\section{- Different designs}

- Micromegas

- 9 mini SAS connectors (8 for FEs, 1 for ADDC)

- $\quad$ STGC

- 3 mini SAS connectors ( 3 for FEs)

- Voltage levels

- 2.5V Digital (VTRX)

- $1.5 \mathrm{~V}$ Analog (GBTX)

- 1.5V Digital (GBTX)

\section{- Components}

- GBTx ASIC, VTRX optical transceiver, 2 x FEAST DC-DC converter

- Power connector - Molex 0015912025

- Power consumption

- 3.5Watts (estimated)

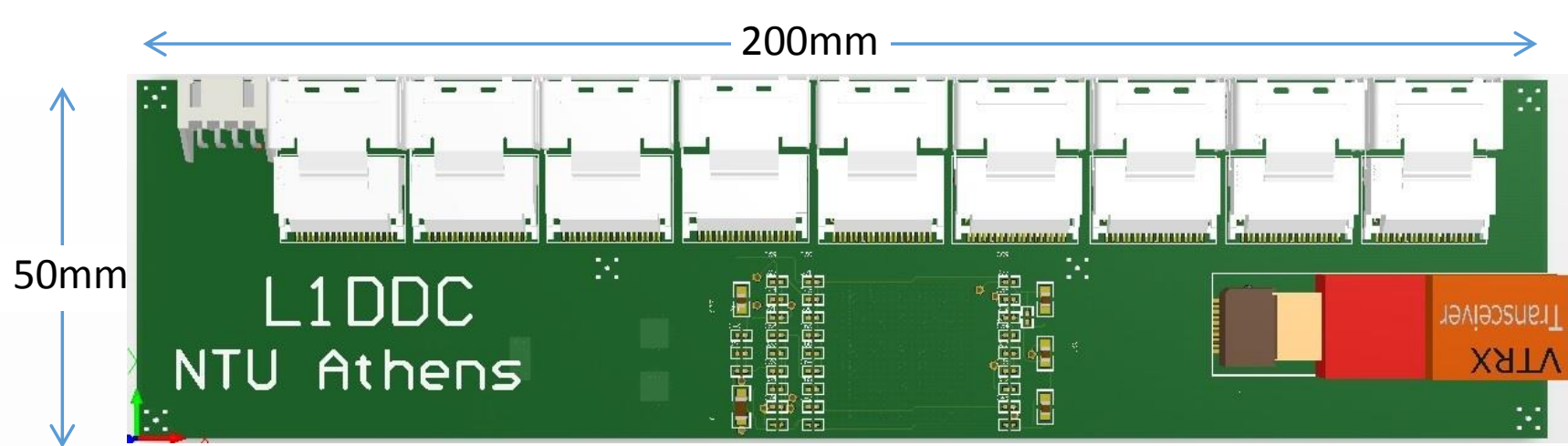

Top side of micromegas L1DDC

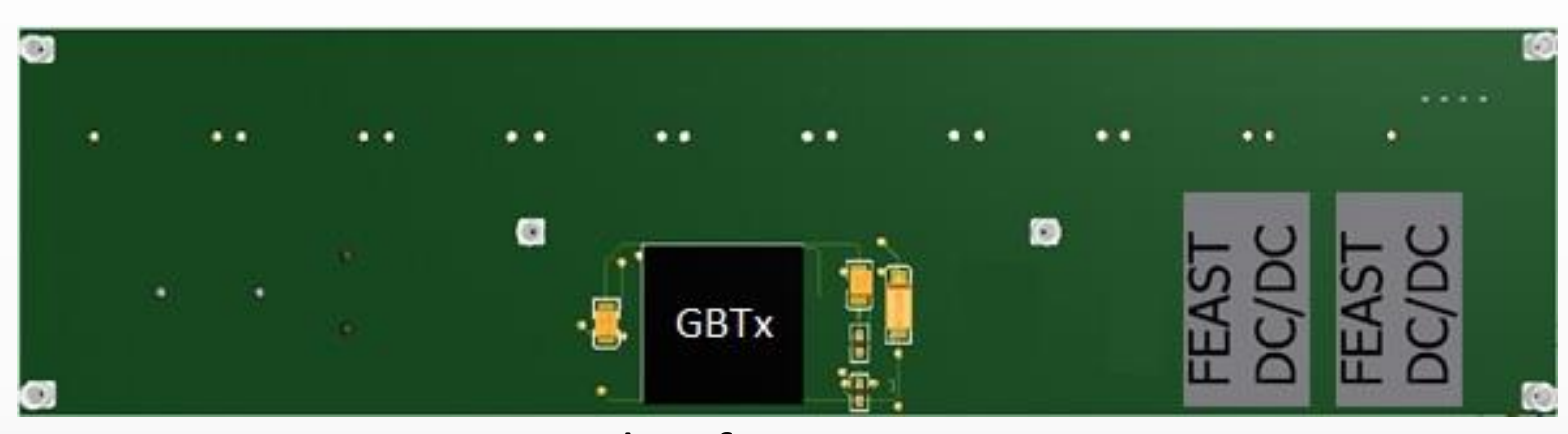

Bottom side of micromegas L1DDC

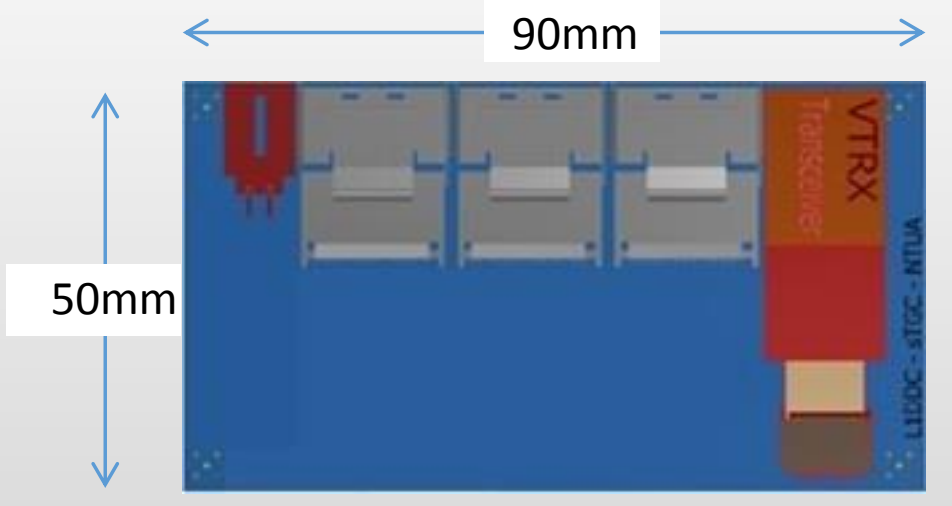

Top side of sTGC L1DDC

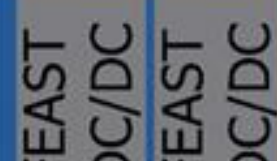

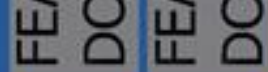

Bottom side of sTGC L1DDC 


\section{L1DDC components}

\section{- GBTX ASIC}

- BGA 400pins package

- Pitch 0.8mm

- Radiation hard ASIC - IBM 130nm technology

- Power supply $1.5 \mathrm{~V}$

- Power consumption 2.2Watts

\section{- VTRX optical transceiver}

- GBTIA

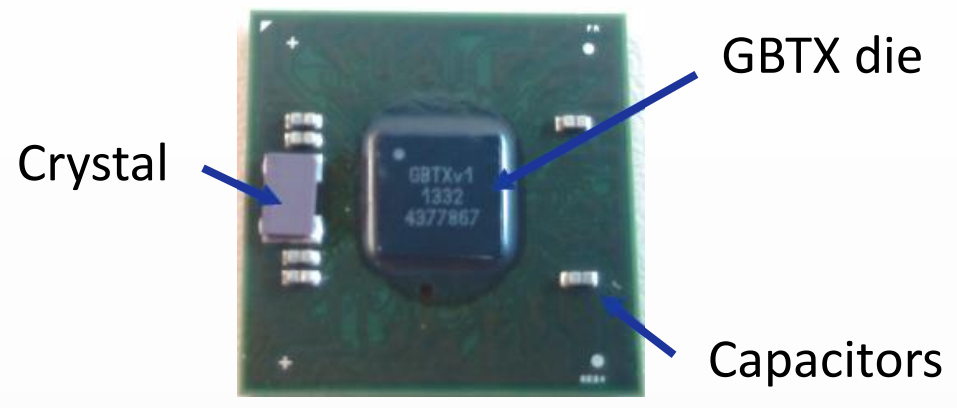

Top side of the GBTX

ASIC

- 2.5V@0.05Amps

- Power consumption 0.12Watts

- Bit rate $5 \mathrm{~Gb} / \mathrm{s}$

- $\quad$ 0.13- $\mu \mathrm{m}$ IBM CMOS8RF-LM technology

- GBLD

- 2.5V@0.2Amps

- Power consumption 0.5Watts

- Bit rate $5 \mathrm{~Gb} / \mathrm{s}$

\section{- FEAST DC-DC converter}

- Input voltage from $5 \mathrm{~V}$ to $11.5 \mathrm{~V}, 4 \mathrm{~A}$ load capacity

- $76 \%$ efficiency

- It contains a radiation tolerant ASIC with total ionizing dose up to

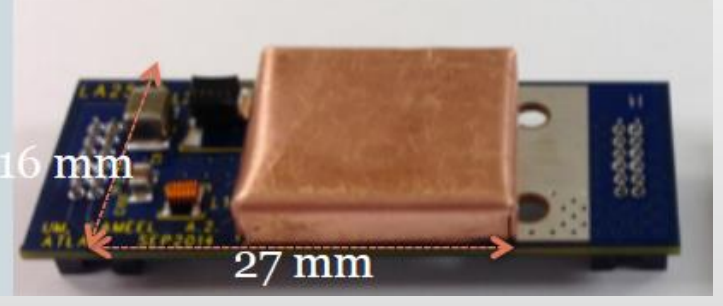

VTRX optical transceiver $200 \mathrm{Mrad}$ (Si) and displacement damage up to $5 \times 10^{14} \mathrm{n} / \mathrm{cm} 2$

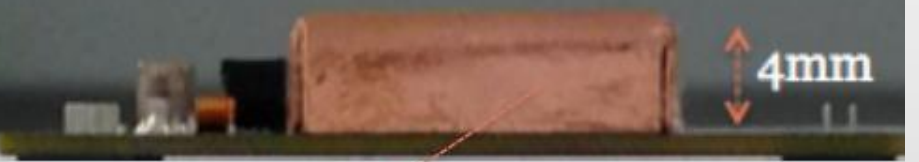




\section{L1DDC placement}
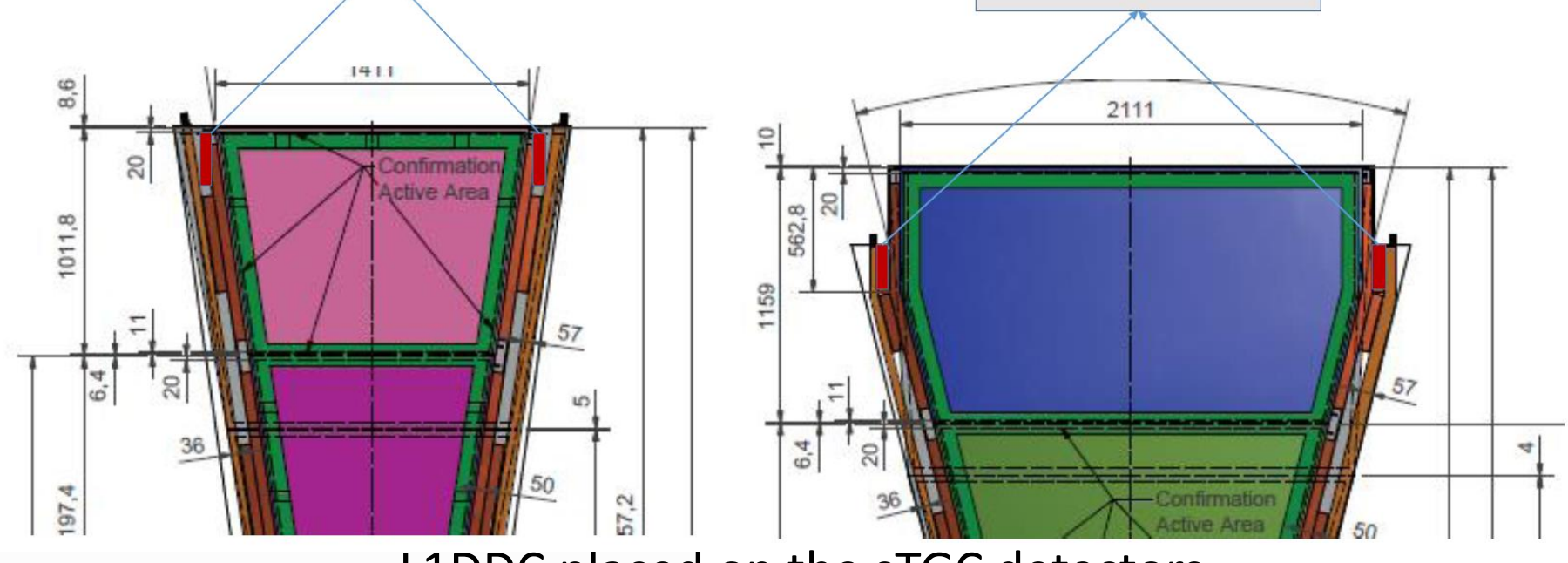

L1DDC placed on the STGC detectors @ $3 \mathrm{~m}$ )

- Elastic thermal foam will be used for better connectivity to the cooling channel
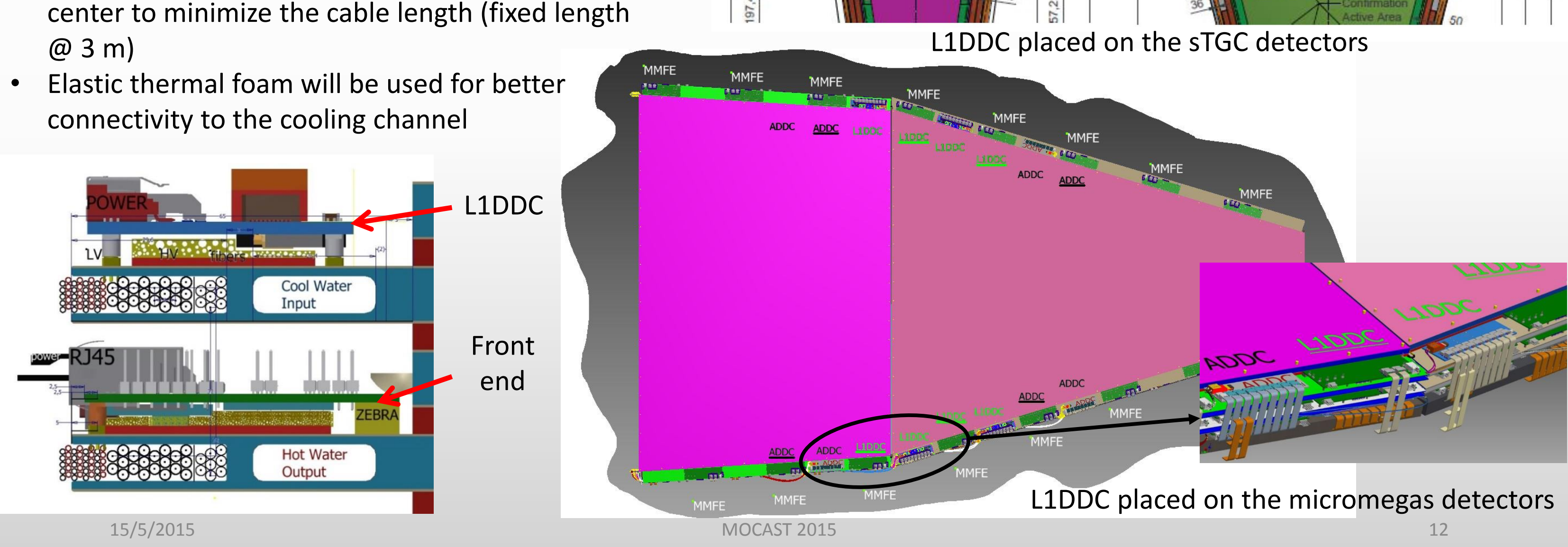


\section{L1DDC - Prototype $1 \& 2$}

\section{- Prototype 1}

- Already fabricated

- Size $210 \mathrm{~mm} \times 144 \mathrm{~mm}$

- Layers used : 14

- Alternative paths in case of GBTX failure

- Input voltage $3.6 \mathrm{~V}-42 \mathrm{~V}$

- GBTx, GBTIA \& GBLD (VTRX) ASICs

- Xilinx FPGA Artix7 - xc7a200t-3FBG484

- SFP+, Gigabit Ethernet, VTRX, miniSAS 36p, SMAs

- Still in debugging process

- Board is functional

- No radiation tolerant board

- Prototype 2

- Design in progress (same as final board)

- Size $200 \mathrm{~mm} \times 50 \mathrm{~mm}$

- 12 Layers will be used

- Input voltage 5V - $12 \mathrm{~V}$

- Use only radiation hard components (GBTx, VTRX, FEAST)

- Can be tested in radiation and magnetic fields

- Estimate final power consumption

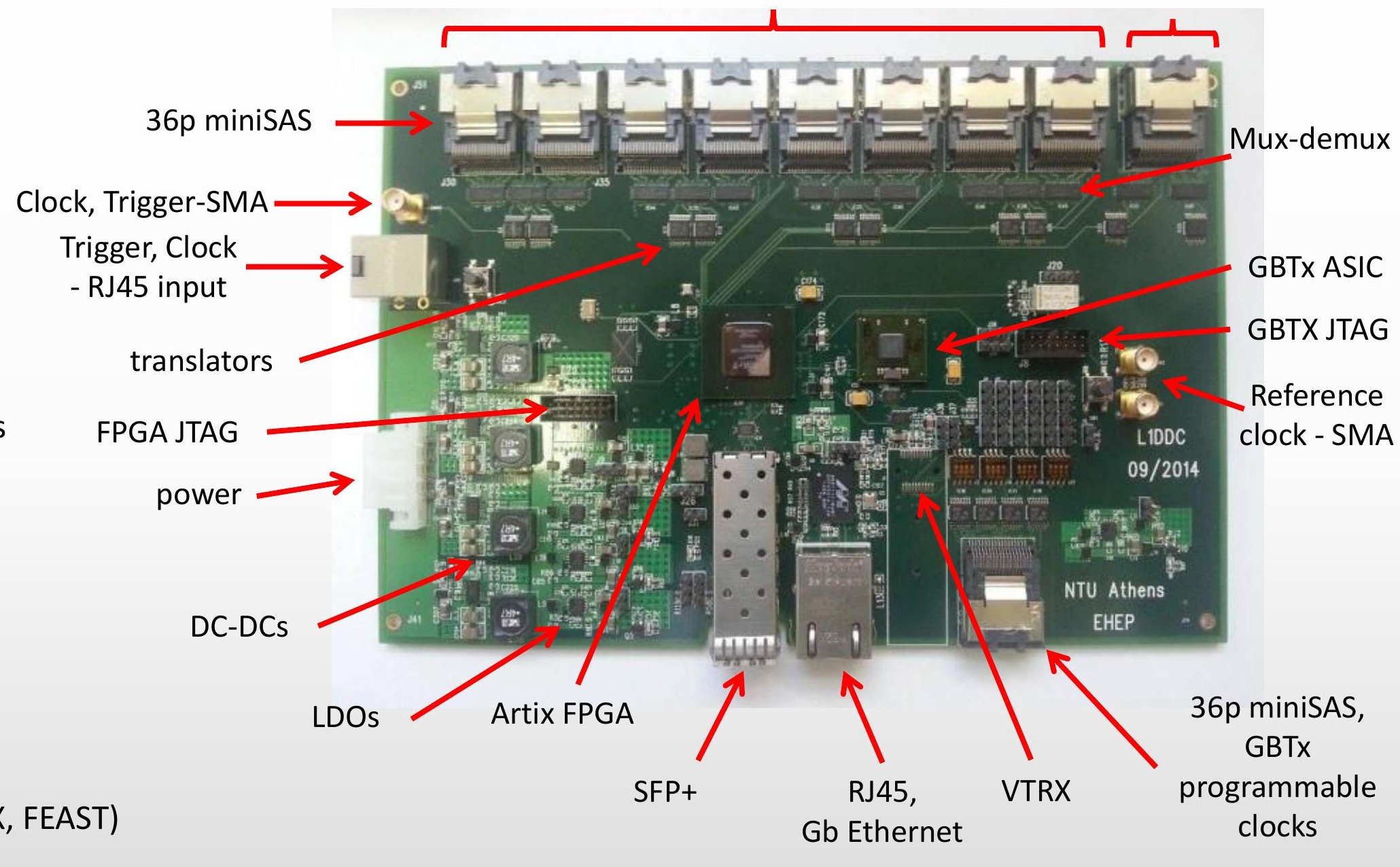




\section{Summary}

- L1DDC collects the Level-1 Data and distributes the TTC data to the front end and ADDC boards

- L1DDC is a fully radiation tolerant board

- Fulfills all ATLAS NSW upgrade requirements

- Fully compliant with LHC rates

- Has SEU mechanisms to assure signal integrity

- Different L1DDC boards will be fabricated for micromegas and STGCs detectors 
Thank you

\section{Questions \&ll}




\section{Backup Slides}




\section{MMs and sTGC rates}
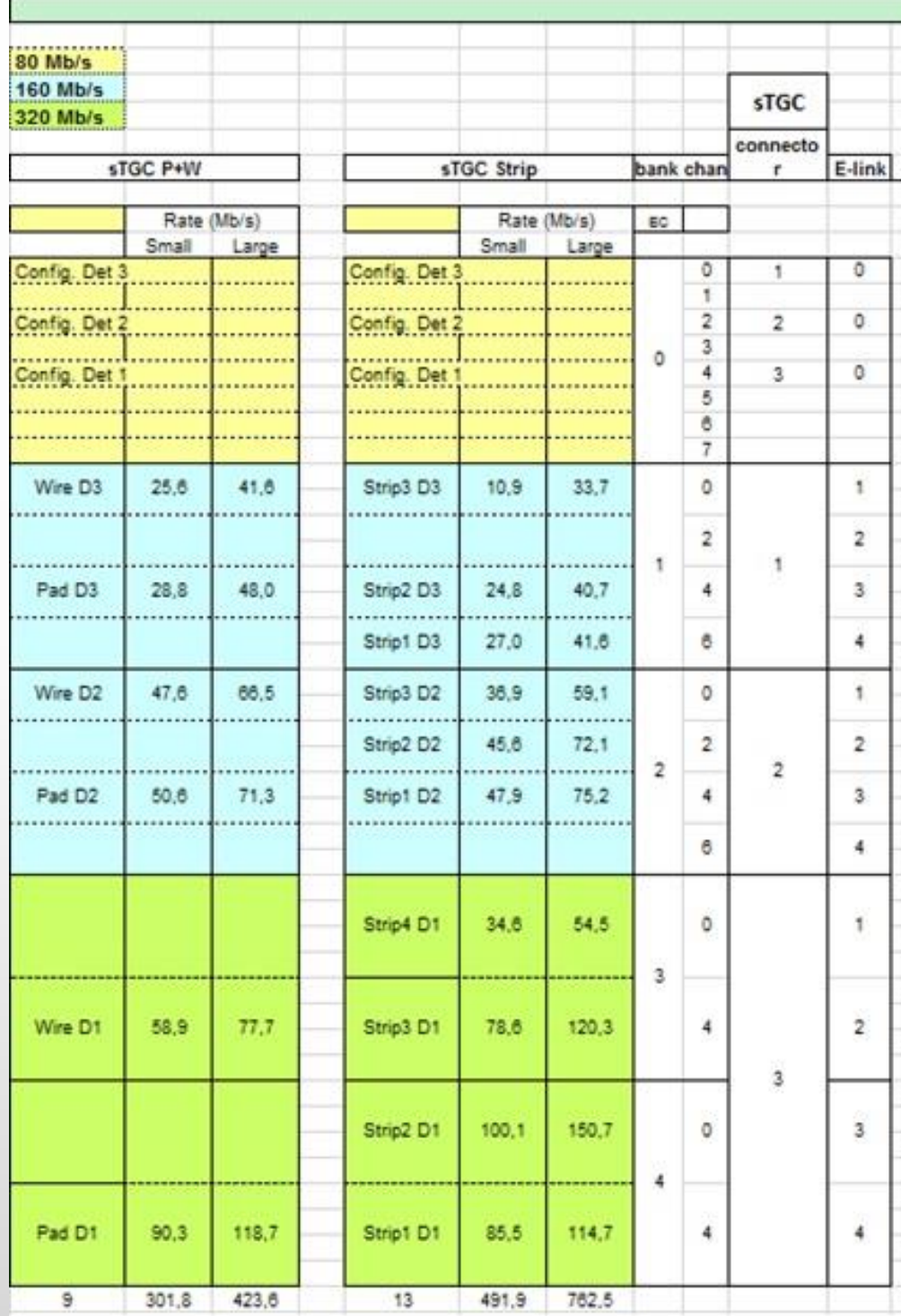

$15 / 5 / 2015$

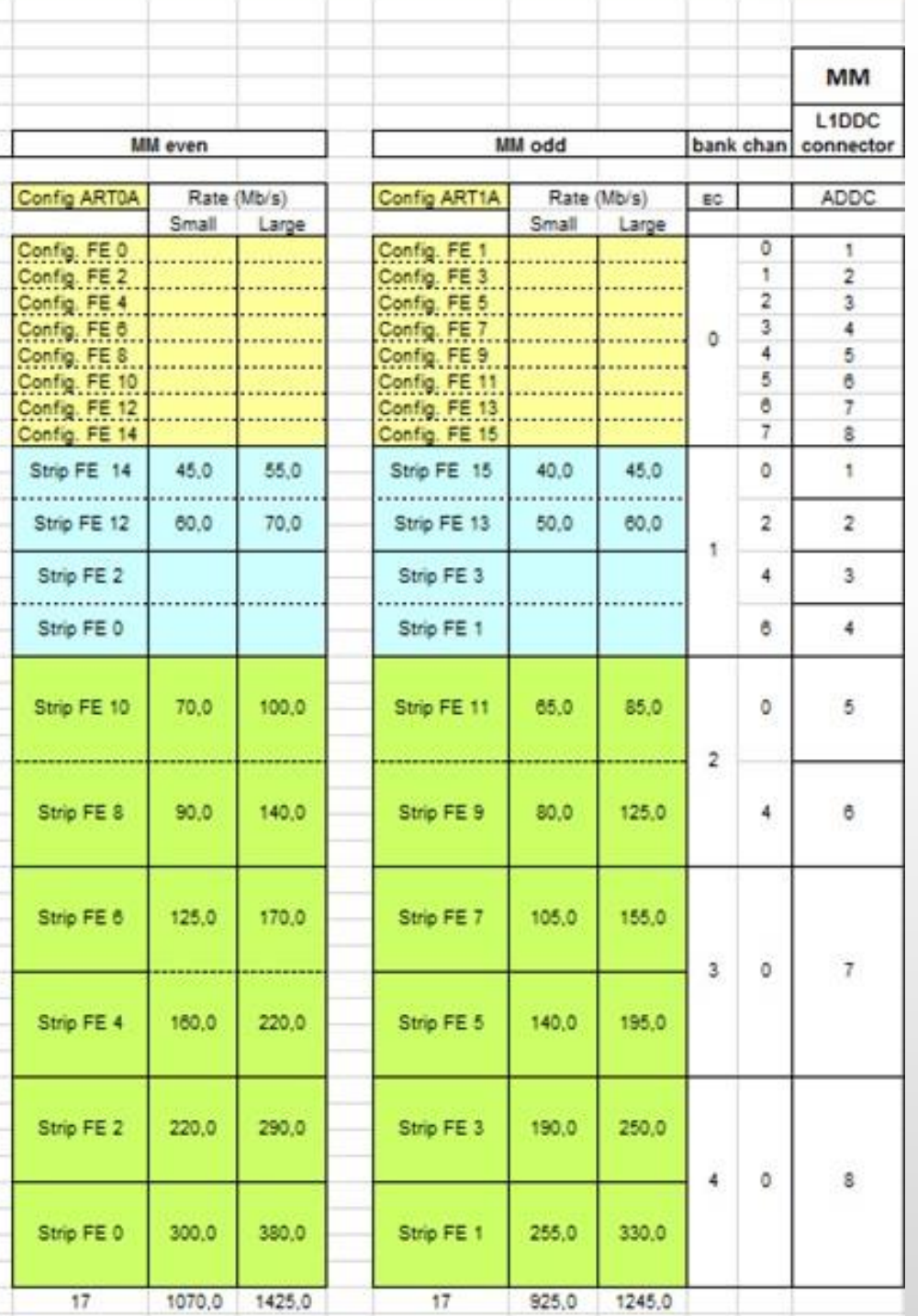

- MMs rates exceed maximum of e-link rate.

- 2 elinks/MMFE8 will be used for the inner portions (1 elink @ 320Mbps+1 elink@160 Mbps = 480Mbps).

- 3 sTGC FEs will be connected to 1 L1DDC (1 twinax cable and 10 differential pairs)

\begin{tabular}{|c|c|c|c|c|c|}
\hline \multicolumn{6}{|c|}{ sTGC twinax for FE board readout to L1DDC-sTGC } \\
\hline pair & & & connection & use & \\
\hline 1 & Elink0 & clock & SCA & config clk & \\
\hline 2 & Elink0 & from GBT & SCA & config & \\
\hline 3 & Elink0 & to GBT & SCA & config & \\
\hline 4 & Elink1 & clock & ROC & ROC clk & \\
\hline 5 & Elink1 & from GBT & ROC & TTC & \\
\hline 6 & Elink1 & to GBT & SROC & L1 data & \\
\hline 7 & Elink3 & to GBT & SROC & L1 data & \\
\hline 8 & Elink3 & to $\mathrm{GBT}$ & SROC & L1 data & \\
\hline 9 & Elink4 & to GBT & SROC & L1 data & \\
\hline 10 & spare & & & spare & \\
\hline
\end{tabular}




\section{L1DDC prototype1 - technical characteristics}

- FPGA Artix 7 (xc7a200t-3fbg484)

- Configures the GBTX

- Implements the I2C protocol for the communication with the configuration registers

- Reads and sends the data in case of GBTx failure

- Inputs \& outputs

- Detector side

- Elinks (LVDS or SLVS differential)

- Counting room side

- VTRX optical tranceiver

- 10/100/1000 ethernet

- SFP

- SMA

- JTAG port

- SMA \& RJ45 for reference clock and trigger 


\section{L1DDC prototype1 - technical characteristics}

- Voltages Levels

- 1.5V Digital (GBTx)

- 1.5V Analog (GBTx)

- 2.5V Digital (VTRx, FPGA) Direct from LTM4619

- 3.3V Digital (efuses, SN65LVDT122) Direct from LTM4619

- 1.2V Analog MGTAVTT (FPGA)

- 1.0V Analog MGTAVCC (FPGA)

- 1.0V Digital VCCINT, VCCBRAM (FPGA)

- 1.8V Digital VCCAUX (FPGA)

- 4 LT8612 DC-DC Converters

- Single 6A output

- Use of 6 ADP1755 LDOs to step down the voltage

- Singe output @ 1.2A

- Anything to LVDS translators (SN65LVDT122)

- Convert GBTx output (SLVS) to LVDS standard 


\section{Layer stack \& differential impendence}

\begin{tabular}{|c|c|c|c|c|c|c|c|}
\hline \multicolumn{4}{|c|}{ PCB Stack Up } & \multicolumn{4}{|c|}{ Impedance } \\
\hline Layer & Type & \multicolumn{2}{|c|}{ Thickness (mil) } & Single ended & $\mathrm{OHM}$ & Differential & $\mathrm{OHM}$ \\
\hline \multicolumn{3}{|c|}{ Top side solder mask } & 0,5 mils & & & & \\
\hline \multirow{2}{*}{$\mathrm{L} 1$} & Top & copper + plating & 1,4 mils & & & $4 / 4 / 4$ mils, $100 \Omega+1-10 \%$ & $97.48 \Omega$ \\
\hline & & prepreg & 4 mils & & & & \\
\hline \multirow[t]{2}{*}{$\mathrm{L} 2$} & & copper & 0,7 mils & & & & \\
\hline & & core & 8 mils & & & & \\
\hline \multirow[t]{2}{*}{$L 3$} & & copper & 0,7 mils & & & $4 / 4 / 4$ mils, $100 \Omega+1-10 \%$ & $99.24 \Omega$ \\
\hline & & prepreg & 8 mils & & & & \\
\hline \multirow[t]{2}{*}{$\mathrm{L} 4$} & & copper & 0,7 mils & & & & \\
\hline & & core & 6 mils & & & & \\
\hline \multirow[t]{2}{*}{$\mathrm{L} 5$} & & copper & 0,7 mils & & & $4 / 4 / 4$ mils, $100 \Omega+1-10 \%$ & $97.85 \Omega$ \\
\hline & & prepreg & 5 mils & & & & \\
\hline \multirow[t]{2}{*}{ L6 } & & copper & 0,7 mils & & & $4 / 4 / 4$ mils, $100 \Omega+1-10 \%$ & $97.85 \Omega$ \\
\hline & & core & 6 mils & & & & \\
\hline \multirow[t]{2}{*}{$L 7$} & & copper & 0,7 mils & & & & \\
\hline & & prepreg & 4 mils & & & & \\
\hline \multirow[t]{2}{*}{$\llcorner 8$} & & copper & 0,7 mils & & & & \\
\hline & & core & 6 mils & & & & \\
\hline \multirow[t]{2}{*}{ L9 } & & copper & 0,7 mils & & & $4 / 4 / 4$ mils, $100 \Omega+1-10 \%$ & $97.85 \Omega$ \\
\hline & & prepreg & 5 mils & & & & \\
\hline \multirow[t]{2}{*}{$L 10$} & & copper & 0,7 mils & & & $4 / 4 / 4$ mils, $100 \Omega+1-10 \%$ & $97.85 \Omega$ \\
\hline & & core & 6 mils & & & & \\
\hline \multirow[t]{2}{*}{ L11 } & & copper & 0,7 mils & & & & \\
\hline & & prepreg & 8 mils & & & & \\
\hline \multirow[t]{2}{*}{ L12 } & & copper & 0,7 mils & & & $4 / 4 / 4$ mils, $100 \Omega+1-10 \%$ & $99.24 \Omega$ \\
\hline & & core & 8 mils & & & & \\
\hline \multirow[t]{2}{*}{$\mathrm{L} 13$} & & copper & 0,7 mils & & & & \\
\hline & & prepreg & 4 mils & & & & \\
\hline \multirow[t]{2}{*}{ L14 } & Bottom & copper + plating & 1,4 mils & & & $4 / 4 / 4$ mils, $100 \Omega+1-10 \%$ & $97.48 \Omega$ \\
\hline & \multicolumn{2}{|c|}{ Bottom side solder mask } & 0,5 mils & & & & \\
\hline \multicolumn{3}{|c|}{ TOTAL } & 90,2 mils & & & & \\
\hline
\end{tabular}




\section{L1DDC functionality}

ART

Front End board connections - 8 FEB/L1DDC

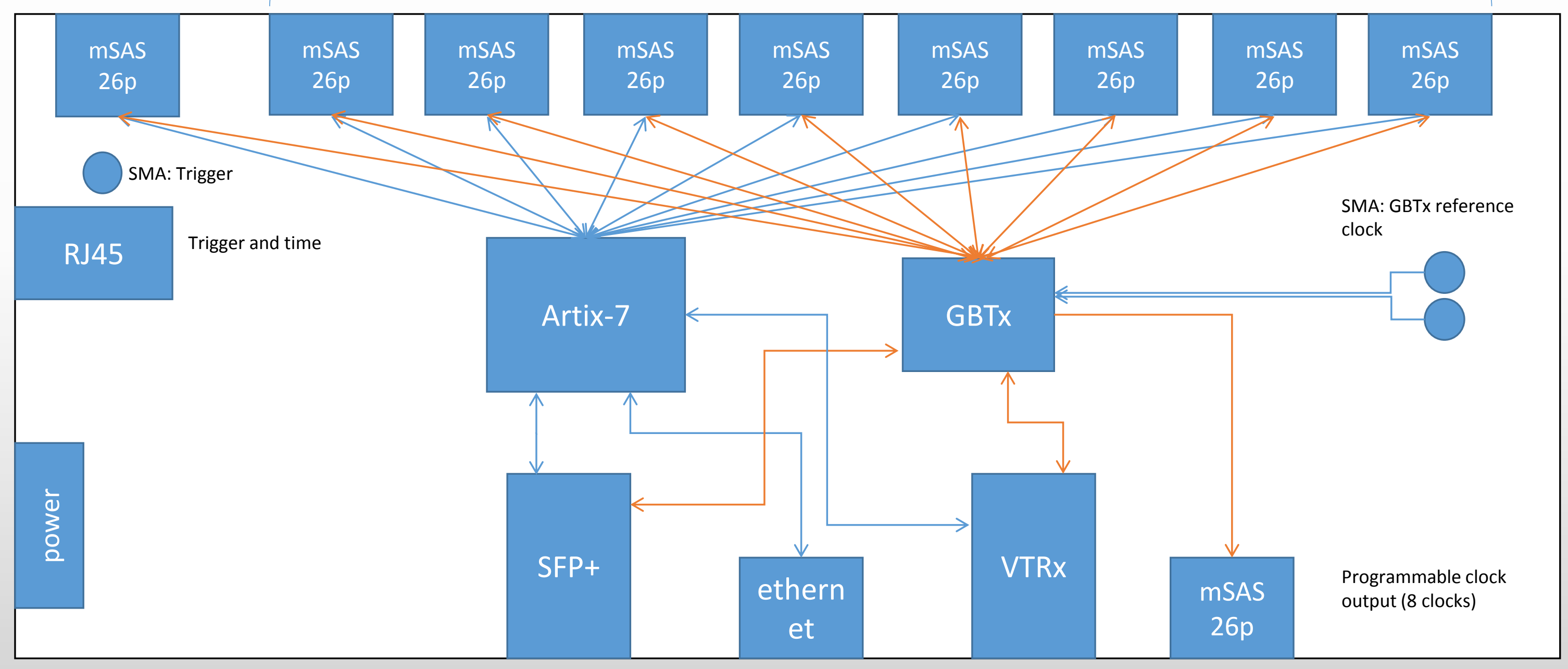




\section{Send \& receive data (FE side)}

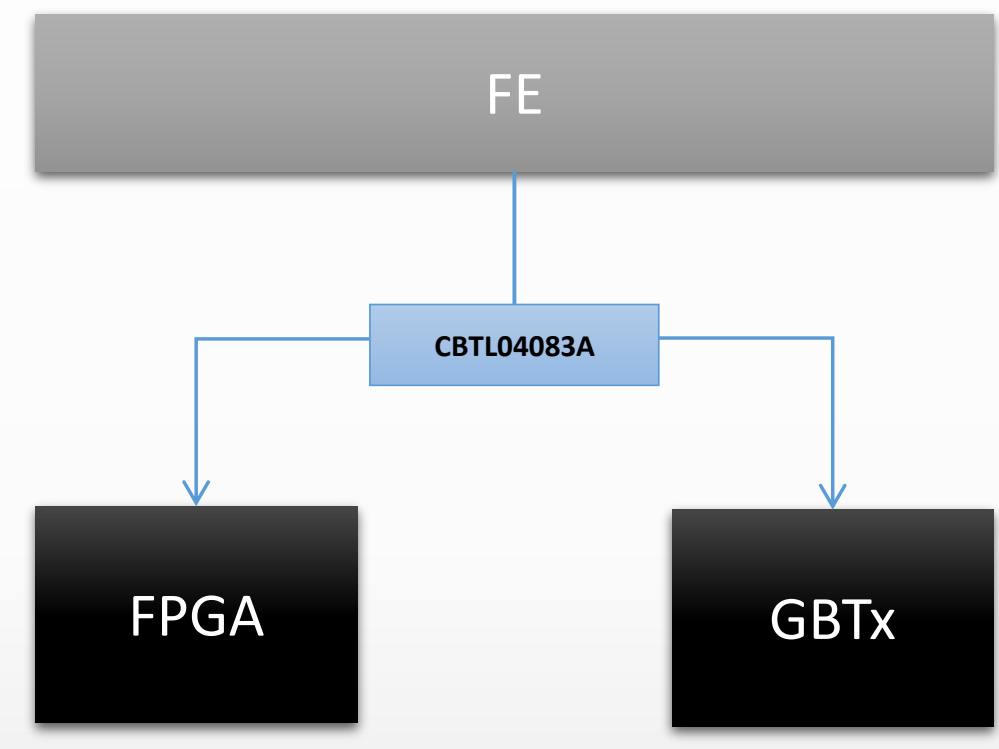

2 alternative paths to read and send the data

- From FPGA in case of failure of GBTX

- Directly from GBTx

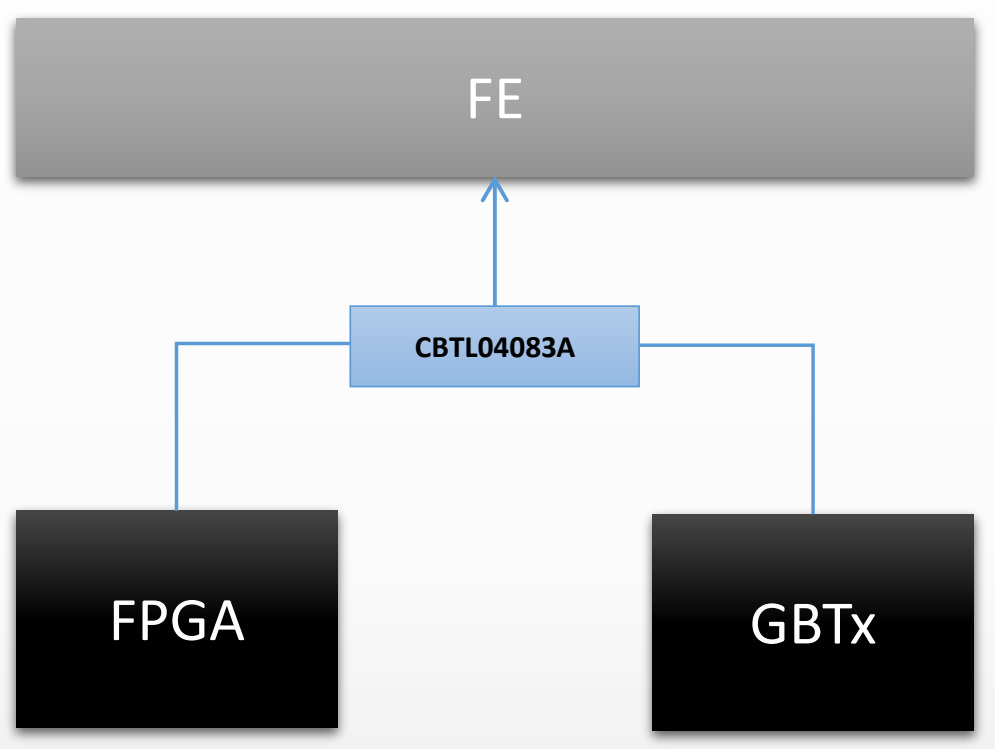

CBTL04083A PCI Express Gen3 switch up to $8.3 \mathrm{~Gb} / \mathrm{s}$

- Low intra-pair skew: 5 ps typical

- Low inter-pair skew: 35 ps maximum 


\section{Connectivity: MMFE8 - L1DDC - ADDC}

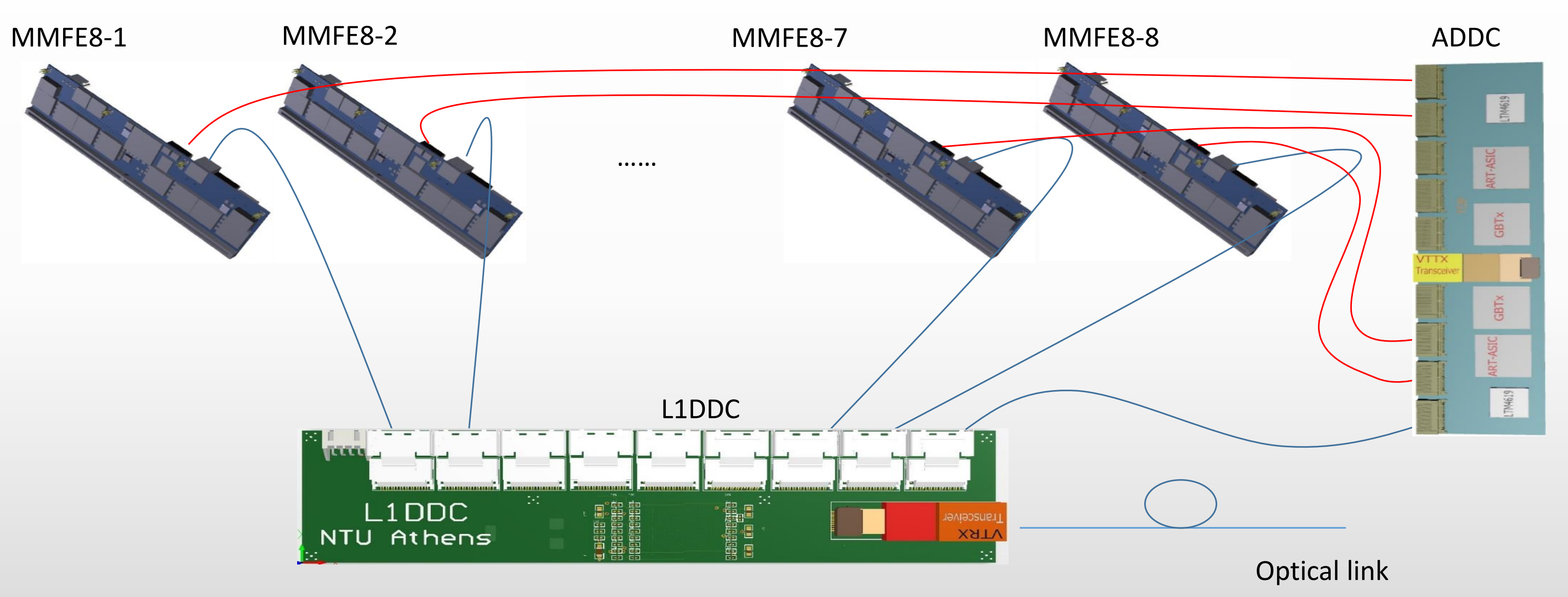




\section{Twinax cables}

- 3M mini SAS cables

- 36p positions

- 8 differential pairs +8 sidebands

- Part No: 8F36-AAA105
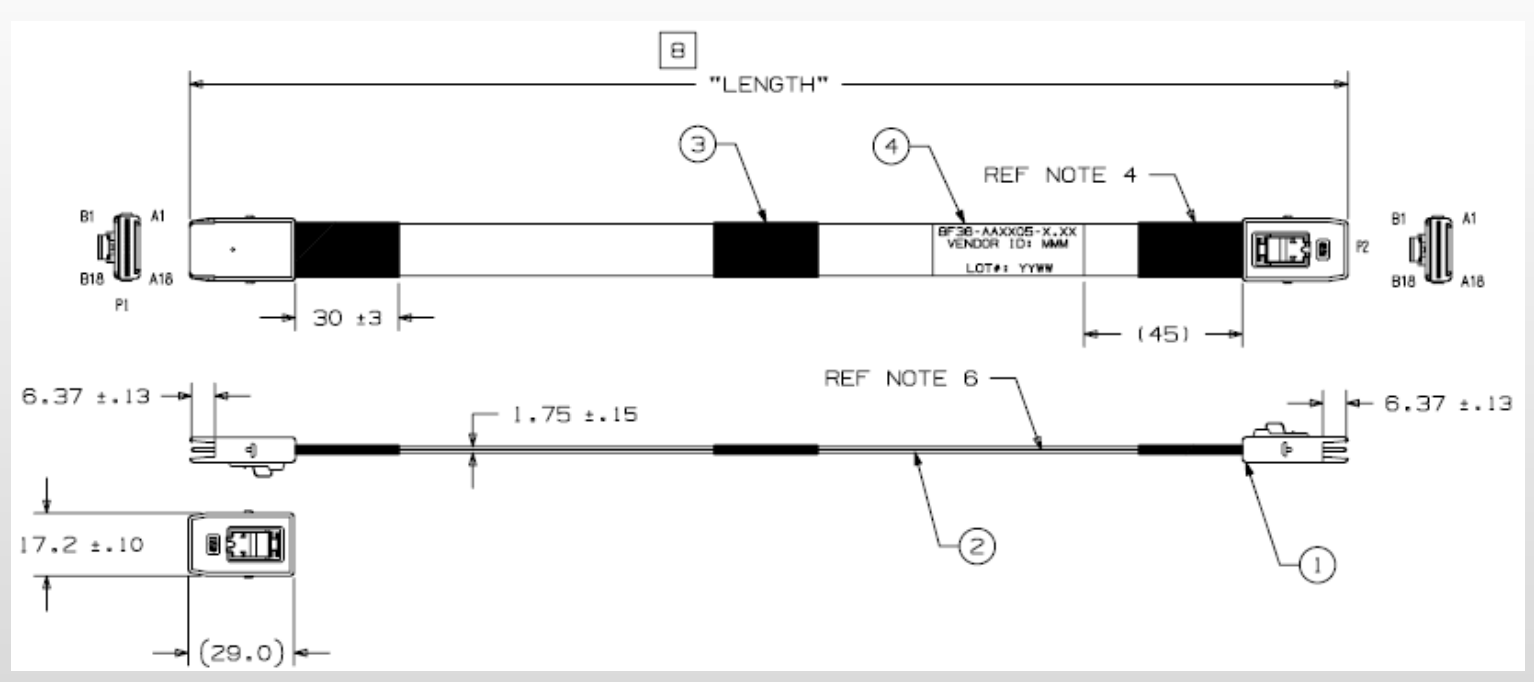

PINOUT 1

BACKPLANE-TO-CONTROLLER

\begin{tabular}{|c|c|c|c|}
\hline \multicolumn{2}{|c|}{ P1 } & \multicolumn{2}{|c|}{ P2 } \\
\hline A1 & GND & B1 & GND \\
\hline A2 & Rx 0+ & B2 & Tx 0+ \\
\hline A3 & Rx 0- & B3 & Tx 0- \\
\hline A4 & GND & B4 & GND \\
\hline A5 & Rx 1+ & B5 & Tx 1+ \\
\hline A6 & Rx 1- & B6 & Tx 1- \\
\hline A7 & GND & B7 & GND \\
\hline A8 & SIDEBAND & B8 & SIDEBAND \\
\hline A9 & SIDEBAND & B9 & SIDEBAND \\
\hline A10 & SIDEBAND & B10 & SIDEBAND \\
\hline A11 & SIDEBAND & B11 & SIDEBAND \\
\hline A12 & GND & B12 & GND \\
\hline A13 & Rx 2+ & B13 & Tx 2+ \\
\hline A14 & Rx 2- & B14 & Tx 2- \\
\hline A15 & GND & B15 & GND \\
\hline A16 & Rx 3+ & B16 & Tx 3+ \\
\hline A17 & Rx 3- & B17 & Tx 3- \\
\hline A18 & GND & B18 & GND \\
\hline
\end{tabular}

\begin{tabular}{|c|c|c|c|}
\hline \multicolumn{2}{|c|}{ P1 } & \multicolumn{2}{c|}{ P2 } \\
\hline B1 & GND & A1 & GND \\
\hline B2 & Tx 0+ & A2 & Rx 0+ \\
\hline B3 & Tx 0- & A3 & Rx 0- \\
\hline B4 & GND & A4 & GND \\
\hline B5 & Tx 1+ & A5 & Rx 1+ \\
\hline B6 & Tx 1- & A6 & Rx 1- \\
\hline B7 & GND & A7 & GND \\
\hline B8 & SIDEBAND & A8 & SIDEBAND \\
\hline B9 & SIDEBAND & A9 & SIDEBAND \\
\hline B10 & SIDEBAND & A10 & SIDEBAND \\
\hline B11 & SIDEBAND & A11 & SIDEBAND \\
\hline B12 & GND & A12 & GND \\
\hline B13 & Tx 2+ & A13 & Rx 2+ \\
\hline B14 & Tx 2- & A14 & Rx 2- \\
\hline B15 & GND & A15 & GND \\
\hline B16 & Tx 3+ & A16 & Rx 3+ \\
\hline B17 & Tx 3- & A17 & Rx 3- \\
\hline B18 & GND & A18 & GND \\
\hline
\end{tabular}

